\title{
Multiscale digital image identification of heterogeneous elastic properties of softwoods
}

\author{
J. Réthoré ${ }^{1, a}$, P. Simon ${ }^{1}$, and H. Maigre ${ }^{1}$ \\ LaMCoS, Université de Lyon / INSA Lyon / CNRS, 20 Avenue Albert Einstein, 69621 Villeurbanne, \\ France
}

\begin{abstract}
Multi-scale modelling of materials is now widely spread as it allows for a comprehensive analysis of their behaviour. The idea of this paper is to propose a methodology that couples Digital Images Correlation with multi-scale numerical simulations in order to identify a material behaviour that is too complex to obtain at the observation scale but that can be described in a simpler way at a finer scale. For this purpose, a Mechanical Image Correlation approach is proposed that involves a multi-scale numerical model. The capabilities of the proposed method is illustrated based on an example dedicated to the identification of heterogeneous orthotropic elastic properties of softwood.
\end{abstract}

\section{Introduction}

Multi-scale modelling of materials is now a common pratice. Indeed, from a complex behaviour at the observation scale, going to finer scales usually leads to simpler and more intrinsic models. The idea has been exploited during the last decades to design multi-scale numerical investigation tools. However, during an experiment the observation scale is usually the modelling scale and also the identification scale.

Digital Image Correlation (DIC) has also gained in the past decades an increased popularity. DIC is one of the most popular full field measurement techniques as it is easy to obtain digital images of the surface of a sample during an experiment. Further, some recent developpements show that DIC can be coupled intimately with numerical simulations as soon as the same basis functions is used to describe the displacement field in both analysis [1].

For identification purpose, various techniques have been proposed based on full field measurements. One can distinguish between direct identification techniques like the Constitutive Equation Gap Method, the Reciprocity Gap Method, the Equilibrium Gap Method, the Virtual Field Method (see [2] for an overview) and Finite Element Model Updating based approaches. The latter was first proposed in [3].

The idea of this paper is to propose a methodology that couples DIC with multi-scale numerical simulations in order to identify a material behaviour that is too complex to obtain at the observation scale but that can be described in a simpler way at a finer scale. For this purpose, a Mechanical Image Correlation (MIC) [4] approach is proposed that involves a multi-scale numerical model. The approach is illustrated based on an example dedicated to the identification of heterogeneous orthotropic elastic properties of softwood.

\section{Multi-Scale model of softwood}

In this study, wood is descripted at different scales, going from the annual ring to the cell. The aim of such a description is to link a quite complex and heterogeneous mechanical behaviour to simple

a e-mail: julien.rethore@insa-lyon.fr

This is an Open Access article distributed under the terms of the Creative Commons Attribution-Noncommercial License 3.0, which permits unrestricted use, distribution, and reproduction in any noncommercial medium, provided the original work is properly cited. 


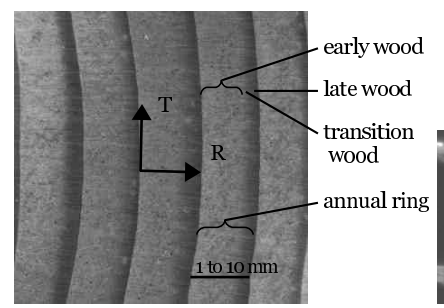

(a)

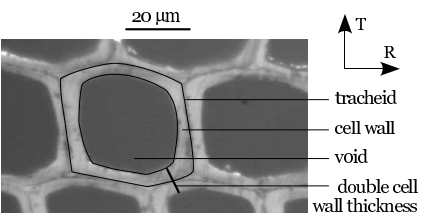

(b)

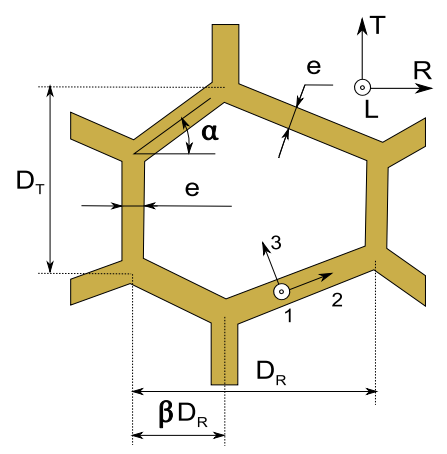

(c)

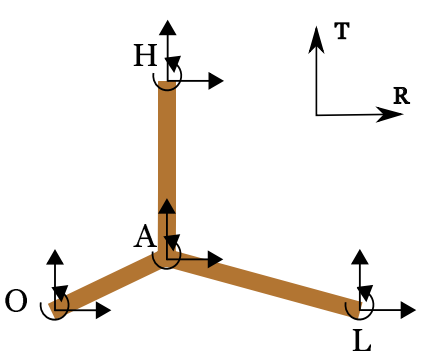

(d)

Fig. 1. Softwood annual rings (a) and cells (b). Modelisation of cells (c): 5 independant parameters: 3 dimensions $D_{R}, D_{T}, e$ and 2 geometric parameters $\alpha$ and $\beta$. (d): morphology of an equivalent cell for mechanical behaviour

material parameters. At the scale of the growth ring, the only material parameter would be the local density. Its space variations are similar to those of mechanical moduli: it varies a lot (from 1 to 4 ) in the radial direction, and quite not in the tangential direction. It is obvious that local stiffness grows with local density, but this only parameter does not explain the local orthotropy of wood. That is why wood has been modelised at the scale of the cells.

\subsection{Wood morphology}

Figure 1 shows the well-known annual or growth rings of a typical softwood in the transverse plane called RT. These rings are constituted of many cells which density varies between the light early-wood and the dense late-wood. These last two zones can be separated by transition wood.

Cells, also called tracheids are stuck to each other and take geometrical but very multiple shapes.

\subsection{Model of the cell}

The examination of the natural structure of softwood cells leads us to the geometrical model given on Figure 1(c). The cell geometry depends on five independant parameters.

Three are dimensions:

- the radial width or diameter $D_{R}$

- the tangential width or diameter $D_{T}$

- the thickness of double cell walls $e$

The last two parameters are geometric indications:

$-\alpha$ gives a measure of the angle between the radial direction and the radial walls.

$-\beta$ indicates the crossing between cells in the radial direction. If the cells are crossed in their middle then $\beta=1 / 2$ and if the tangential walls are aligned, $\beta=0$. In this last case, $\alpha=0$. 
This geometric description of the cell is used to estimate the mechanical behaviour of the cellular material which is softwood. In order to calculate a local behaviour, a cell geometry is fixed (five values for the five parameters). Then, the lattice of cells is artificially considered as infinite. Thanks to this geometrical periodicity and the symetries, the calculation is made on a small part of the lattice. See Figure 1(d) as an illustration. Here cell walls are considered as beams. The model needs the use of the transverse modulus of cell walls in direction $2, E_{2}$ and their shear modulus $G_{23}$ in the transverse plane. See Figure 1(c) for directions 2 and 3.

Then, from the seven values of $D_{R}, D_{T}, e, \alpha, \beta, E_{2}$ and $G_{23}$, one can deduce the elastic behaviour of the coresponding infinite celular material in the transverse plane $R T$. This behaviour will be here caracterized by the four moduli $C_{R R R R}, C_{T T T T}, C_{R R T T}=C_{T T R R}$ and $C_{R T R T}$.

The hypothesis of an infinite latice is not so strong, because only the first neighbouring cells actually influence the behaviour of a cell, and because in softwoods, the morphologie of cells does not change rapidly from cell to cell.

It would be very long, but also useless to try to identify the seven parameters and their spatial variations. Indeed, they are not all completly independant and simplifications can be done.

- Dimensions and moduli are quite constant in the tangential direction.

- The tangential width $D_{T}$ does not vary in the radial direction and does not vary much from a softwood specie to another. Its mean value is in the range of [30 $\mu \mathrm{m} ; 50 \mu \mathrm{m}]$.

- The radial width $D_{R}$ and wall thickness $e$ vary a lot from early wood to late wood. But the variation is regular and when $e$ grows, $D_{R}$ decreases. Some measurements on spruce morphology allow us to link $e$ and $D_{R}$ by the relationship: $D_{R}=60 * e^{-0.12 * e}$

Concerning $\alpha$ and $\beta$, they vary randomly both in radial and tangential directions. To simplify the use of the cellular model, homogeneization has been used to determine the behaviour of a cellular structure with random $\alpha$ and $\beta$. The corresponding elastic moduli can be approached with an equivalent cell morphology. This configuration, with $\beta=0.18$ and $\alpha=21^{\circ}$ is given on Figure 1(c).

The stiffness of cell walls is due to their complex microstructure. It varies a little bit between early and late wood but it can be considered as constant. $G_{23}$ is approximatively the third of $E_{2}$ but this relationship depends on the proportion of the constituants of cell walls.

\section{Identification procedure}

From two grey level images $f$ and $g$ of a sample, DIC consists in a nonlinear minimization problem to identify the displacement field $\mathbf{u}$ that produces the advection of the local texture:

$$
f(\mathbf{x})=g(\mathbf{x}+\mathbf{u}(\mathbf{x}))
$$

To circumvent the illposedness of the problem, it is solved in average over a Region Of Interest (ROI) throught the minimization of the following functional:

$$
\Phi=\{\mathbf{F}-\mathbf{G}(\mathbf{u})\}^{T}\{\mathbf{F}-\mathbf{G}(\mathbf{u})\}=\sum_{p \in R O I}\left[f\left(\mathbf{x}_{\mathbf{p}}\right)-g\left(\mathbf{x}_{\mathbf{p}}+\mathbf{u}\left(\mathbf{x}_{\mathbf{p}}\right)\right)\right]^{2}
$$

where $p$ is the indice of a pixel within the ROI, $\mathbf{x}_{\mathbf{p}}$ the co-ordinates of pixel $p$ on the frame of $f$ and capital letters (e.g. F) denote vectors that collects the values of a discrete function $(e . g . f)$ at the pixel $p$ of the ROI. Suppose now that the displacement is decomposed over a basis functions (e.g. finite element [5]), $\mathbf{u}$ is written as:

$$
\mathbf{u}(\mathbf{x})=\sum_{k \in \mathcal{N}} u_{k} \mathbf{N}_{k}(\mathbf{x})=[\mathbf{N}] \mathbf{U}
$$

where $\mathcal{N}$ is the set of finite element degrees of freedom and $\mathbf{N}_{k}$ finite element shape functions. DIC consists in minimizing $\Phi$ with respect to the displacement degrees of freedom $u_{k}$. In the context of material identification, the sought variable are the set of the material parameters $\boldsymbol{\Lambda}$ that describe the material behaviour. Thus, the set of displacement degrees of freedom is not of direct interest, the 
unknown with respect to which one may minimize $\Phi$ are the material parameters $\boldsymbol{\Lambda}$. In [4] and [6], Mechanical Image Correlation (MIC) is thus formulated as follows: given $f$ and $g$ and a material model, we search for the parameters $\bar{\Lambda}$ that minimize $\Phi$ :

$$
\mathbf{\Lambda}=\operatorname{Arg} \operatorname{Min}\{\mathbf{F}-\mathbf{G}(\mathbf{\Lambda})\}^{T}\{\mathbf{F}-\mathbf{G}(\mathbf{\Lambda})\}
$$

If an iterative procedure is run, assuming small solution increments $\mathrm{d} \boldsymbol{\Lambda}, \Phi$ is linearized and $\mathrm{d} \boldsymbol{\Lambda}$ is obtained by solving the following linear system:

$$
[\mathbf{M}]\{\mathrm{d} \mathbf{\Lambda}\}=\{\mathbf{b}\}
$$

where

$$
[\mathbf{M}]=\left[\frac{\Delta \mathbf{U}}{\Delta \mathbf{\Lambda}}\right]^{T}[\mathbf{N}]^{T}[\nabla \mathbf{G}][\nabla \mathbf{G}][\mathbf{N}]\left[\frac{\Delta \mathbf{U}}{\Delta \mathbf{\Lambda}}\right]
$$

and

$$
\{\mathbf{b}\}=\left[\frac{\Delta \mathbf{U}}{\Delta \mathbf{\Lambda}}\right]^{T}[\mathbf{N}]^{T}[\nabla \mathbf{G}]\{\mathbf{F}-\mathbf{G}(\mathbf{\Lambda})\}
$$

In thoses equations, $\nabla$ denotes spatial differenciation of the deformed image $g$, $\frac{\Delta \mathbf{U}}{\Delta \Lambda}$ is the matrix that express the sensitivity of $\mathbf{U}$ to each material parameter. In pratice, $\mathbf{U}$ is obtained by a multi-scale numerical simulation using a given set of material parameters and appropriate boundary conditions.

\section{Shear test}

\subsection{Test description}

As shown by Figure 2, two pieces of Douglas-fir cut within a ring are glued onto metal plates. This test rig is placed in a tensile machine so that a shear loading is applied to the wood samples. Due to test imperfections and softwood heterogeneities, the test cannot be analyzed as a pure shear material test but structural effect are expected. Figure $2 b$ shows the deformed mesh of a DIC analysis (between the reference image shown by Figure $2 \mathrm{a}$ and a deformed image taken when a shear load of $F o=312 \mathrm{~N}$ has been applied) over the entire test rig using 32-pixel elements. The expected kinematics is obtained but the magnification of the deformation allows one to observe that the bottom and top metal plates do not remain parallel. In this context, the proposed methodology is even more attractive.

A sprayed black and white pattern is applied on the surface of the sample in order to feed DIC algorithm properly. The specimen thickness is $25 \mathrm{~mm}$ and two ROIs (ROI1 and ROI2) are analyzed. Each ROI has the following dimensions: $675 \times 195$ pixels. These ROIs have been positioned approximately over the area made of early wood. The pixel size is $30 \mu \mathrm{m}$ what is about the cell width and thus leads to a large enough scale separation. Indeed, 8-pixel elements will be used in the sequel what is about one order of magnitude larger than the typical cell width.

The aim of this analysis is at identifying the heterogeneous orthotropic elastic moduli of early wood at the observation scale (i.e. the scale of the ring) by using a multi-scale identification procedure that invokes a cell model as described in the previous section. The four moduli $C_{R R R R}, C_{T T T T}, C_{R R T T}=$ $C_{T T R R}$ and $C_{R T R T}$ and their spatial variations are derived from the variation of the cell wall thickness $e$, the cell width in the transverse direction $D_{T}$ and the ratio between the G23 and E2. For the MIC analysis the parameters to identifying are the coefficient that defines the linear variation of $e$ along the radial $(y)$ direction:

$$
\mathbf{\Lambda}=\left\{\lambda_{i}\right\} \operatorname{suchthate}(y)=\sum_{i} l_{i}(y) \lambda_{i}
$$

where $l_{i}$ are unidimensional piecewise linear function supported by a 32-pixel discretization. 


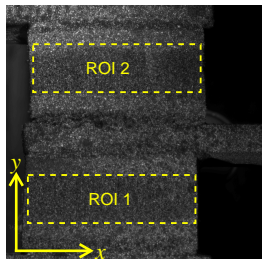

(a)

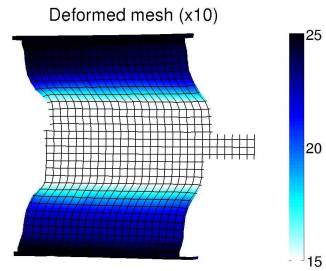

(b)

Fig. 2. ROI1 and ROI2 on the reference image $f$ (a). Displacement norm in pixel plotted on the deformed mesh (amplification factor 10) for a correlation using 16-pixel finite element on the entire test rig.

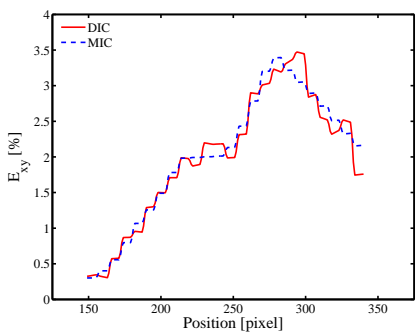

(a)

Fig. 3. Shear strain in percent over ROI 1 for the DIC analysis using 8-pixel elements, the MIC analysis using the same mesh: comparison of the shear strain averaged over the ROI width for the two analyses.

\subsection{Kinematic analysis}

First the analysis is carried out for $D_{T}=41 \mu \mathrm{m}$ and G23=0.35 E2. In Figure 3, we compare the shear strain field obtained with a DIC analysis using 8-pixel elements and the one obtained with a MIC analysis using the same mesh. For the MIC analysis the direct output of the analysis is not displacement but identified material parameters. For visualization purposes, we plot the displacement that is obtained with the identified parameters and the boundary conditions used in the MIC analysis. In the present case, the displacement is prescribed on the top and bottom edges of the ROIs. The values of the prescribed displacement are obtained from a DIC analysis using 32-pixel elements. For comparison, the shear strain field from DIC and MIC have been averaged along the transverse direction $(x)$ and plotted on Figure 3. A good matching is obtained, what confirms the results presented above concerning displacement fields and error maps. These strain maps reveal that the maximum shear strain is not obtained at the begining of early wood ( at the radial location $y$ of about 340 pixels from the bottom of the image) but shifted of about one third of the early wood zone width.

\subsection{Identification results}

Figure 4 confirms that the largest variations of the cell wall thickness (and thus elastic moduli) are obtained within the last third of the early wood zone: $e$ is increasing from 5 to $9 \mu \mathrm{m}$. In the rest of the early wood zone an approximately constant value of $5 \mu \mathrm{m}$ is obtained leading to a cell width in the radial direction around $D_{R}=35 \mu \mathrm{m}$. Note that a perturbation analysis can be carried out for the MIC algorithm [4] and that a mean sensitivity to image noise of $0.035 \mu \mathrm{m}$ per grey level is obtained. The fluctuations of $e$ around $5 \mu \mathrm{m}$ are of about $0.3 \mu \mathrm{m}$ amplitude and thus cannot be explained by noise sensitivity only. Actual variations of the cell wall thickness can thus be appreciated with a very fine resolution (assumed that the proposed modelling is appropriate). Figure $4 \mathrm{~b}$ shows the variations of the elastic moduli that have been identifed by the MIC analysis. At this point of the analysis only stiffness 


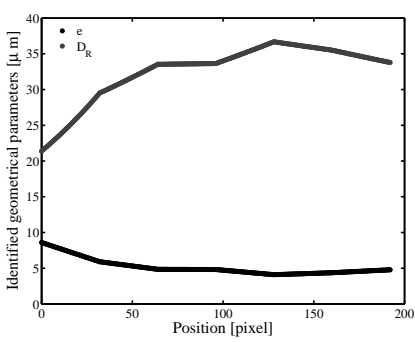

(a)

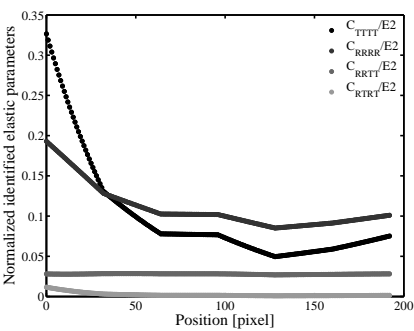

(b)

Fig. 4. Cell morphology parameters $e$ and $D_{R}$ and corresponding identified normalized moduli for G23=0.35E2 and $41 \mu \mathrm{m}$-width cells.

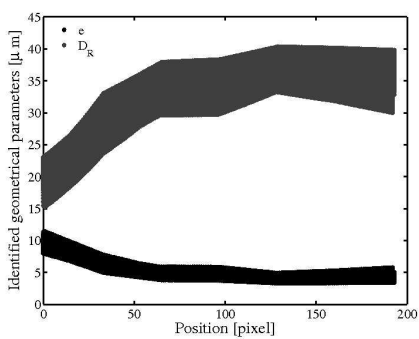

(a)

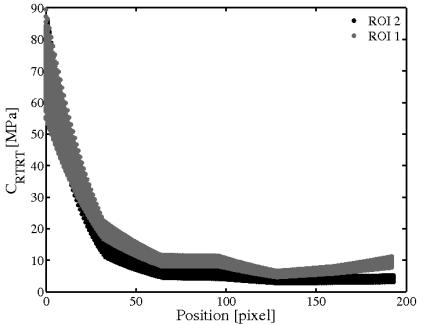

(b)

Fig. 5. Cell morphology parameters $e$ and $D_{R}$ and corresponding identified shear modulus for varying G23/E2 ratio and cell width $D_{T}$.

contrast can be identified as any static data has been used. Hence, the elastic moduli are normalized by E2 the transverse modulus of cell walls. The first observation is that the coefficient corresponding to Poisson's effect $\left(C_{R R T T}\right.$ or $\left.C_{T T R R}\right)$ is almost independent from the cell morphology and thus does not really depends on the position. Conversely, the radial $C_{R R R R}$, transverse $C_{T T T T}$ and shear $C_{R T R T}$ stiffness are strongly affected by cells morphology. $C_{T T T T}$ varies from $1 / 20$ to $1 / 3$ of E2 (from early wood to transition wood), $C_{R R R R}$ from $1 / 10$ to $1 / 5$ and $C_{R T R T}$ from $1 / 1000$ to $1 / 100$.

As a natural material, wood may be affected by high variability. We thus carried out MIC analyzes for cell wall transverse width $D_{T}$ varying from 25 to $55 \mu \mathrm{m}$ and stiffness ratio G23/E2 varying from 0.2 to 0.5 . Further, the two ROIs are analyzed and the load unbalanced due to test imperfections and quantitative moduli are obtained. Indeed, in the MIC analysis natural boundary conditions are applied to the bottom and top edges of the ROIs. The resulting shear forces are computed, compared one to each other and with the load obtained from the load cell of the tensile machine. The ratio between the F2 and F1 (resulting shear force on ROI1 and ROI2 respectively) allows to appreciate the load unbalance due to test imperfections and the sum of F1 and F2 compared to Fo (load cell data) allows to identify the correct value of E2. As an example for the analysis detailed above (with $d t=41 \mu \mathrm{m}$ and $\mathrm{G} 23=0.35 \mathrm{E} 2$ ), $\mathrm{F} 2 / \mathrm{F} 1$ is about 0.8 and the identified value of $\mathrm{E} 2$ is $6.1 \mathrm{GPa}$ (what is in good agreement with the values that one finds in the literature [7]).

Figure 5 shows the identified cell morphology and shear moduli $C_{R T R T}$ for varying stiffness ratio and $D_{T}$. Whereas the explored range is quite large, well defined variations of both morphological parameters and homogenized stiffness are obtained. Indeed, variations with respect to the position relative to the supposed begining of late wood remains the same as described above from Figure 4 except that a relative deviation of \pm 0.3 is obtained for $e$. This leads to deviations of \pm 0.175 , respectively \pm 0.2 , for $D_{R}$, respectively $C_{R T R T}$ which finally increases in average from $15 \mathrm{MPa}$ to $75 \mathrm{MPa}$ in the radial direction. 


\section{Conclusion}

This paper proposes a methodology that allows to identify directly from digital images material properties that arise from a scale finer than the observation scale. Indeed, using a multi-scale numerical simulation embedded into a Mechanical Image Correlation technique, we were able to identify microscopic morphological properties of a wood sample from which we also obtained, through the multiscale model, the macroscopic heterogeneous orthotropic elastic properties.

This paper is a first attempt to integrate a multi-scale model into an identification / measurement technique using digital images. Unless the homogenization we use in the present work is pretty crude, the capabilities of such strategy have been demonstrated. In a near future, more complex multi-scale models will be incorporated in $2 \mathrm{D}$ but also in $3 \mathrm{D}$ analysis using $\mu$-tomographic images as the extension of the present approach to 3D is straightforward.

\section{References}

1. J. Rannou, N. Limodin, J. Réthoré, A. Gravouil, W. Ludwig, M. Baietto-Dubourg, J. Buffière, A. Combescure, F. Hild, S. Roux, Computer Methods in Applied Mechanics and Engineering (2009)

2. S. Avril, M. Bonnet, A. Bretelle, M. Grediac, F. Hild, P. Ienny, F. Latourte, D. Lemosse, S. Pagano, E. Pagnacco et al., Experimental Mechanics 48(4), 381 (2008)

3. K. Kavanagh, R. Clough, International Journal of Solids and Structures 7, 11 (1971)

4. J. Réthoré, International Journal for Numerical Methods in Engineering (submitted)

5. G. Besnard, F. Hild, S. Roux, Experimental Mechanics 46(6), 789 (2006)

6. H. Leclerc, J. Perie, S. Roux, F. Hild, Computer Vision/Computer Graphics CollaborationTechniques (Springer, Berlin, 2009), chap. Integrated Digital Image Correlation for the Identification of Mechanical Properties

7. L. Salmén, Micromechanics of the wood cell wall: a tool for a better understanding of its structure, in First International Conference of the European Society for Wood Mechanics (2001) 